\title{
Multifocal Annular Pink Plaques With a Central Violaceous Hue
}

Reba Suri, MD; Wendy Kim, DO

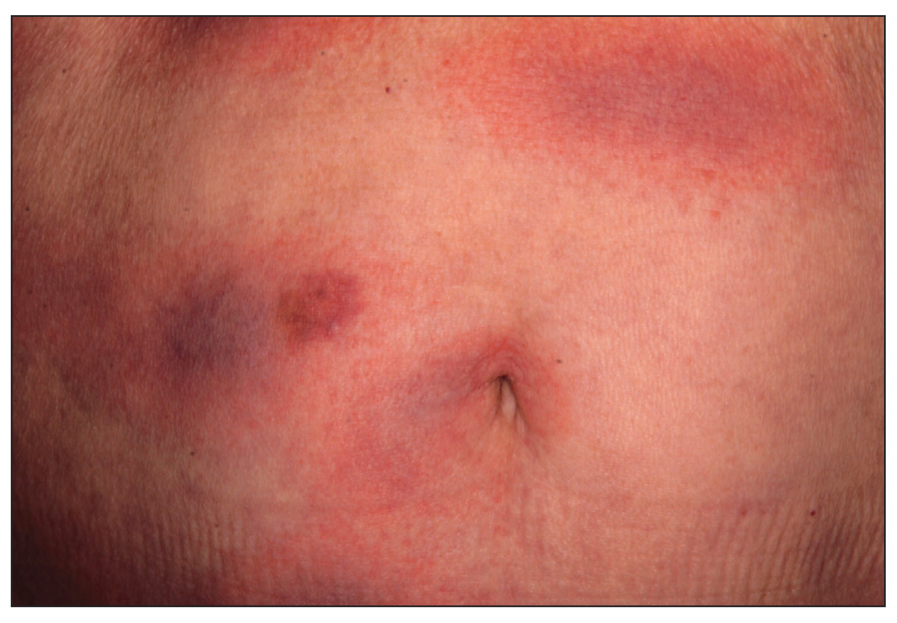

An otherwise healthy 78-year-old woman presented with a diffuse, mildly itchy rash of 5 days' duration with associated fatigue, chills, decreased appetite, and nausea. She reported waking up with her arms "feeling like they weigh a ton." She denied any pain, bleeding, or oozing and was unsure if new spots were continuing to develop. The patient reported having allergies to numerous medications but denied any new medications or recent illnesses. She had recently spent time on a farm in Minnesota, and upon further questioning she recalled a tick bite 2 months prior to presentation. She stated that she removed the nonengorged tick and that it could not have been attached for more than 24 hours. Her medical and family history were unremarkable. Physical examination showed multiple annular pink plaques with a central violaceous hue in a generalized distribution involving the face, trunk, arms, and legs with mild erythema of the palms. The plantar surfaces were clear, and there was no evidence of lymphadenopathy. The remainder of the physical examination and review of systems was negative. Laboratory screening was notable for an elevated erythrocyte sedimentation rate and C-reactive protein level with negative antinuclear antibodies.

\section{WHAT'S YOUR DIAGNOSIS?}
a. disseminated erythema chronicum migrans
b. erythema multiforme
c. fixed drug eruption
d. Sweet syndrome
e. urticaria

Dr. Suri is from the School of Medicine, University of Nevada, Reno. Dr. Kim is from the Dermatology Department, Loyola University Medical Center, Maywood, Illinois.

The authors report no conflict of interest.

Correspondence: Reba Suri, MD (suri.reba@gmail.com).

doi:10.12788/cutis.0187 


\section{THE DIAGNOSIS:}

\section{Disseminated Erythema Chronicum Migrans}

E mpiric treatment with doxycycline $100 \mathrm{mg}$ twice daily for 14 days was initiated for suspected early disseminated Lyme disease manifesting as disseminated multifocal erythema chronicum migrans (Figure). Lyme screening immunoassay and confirmatory IgM Western blot testing subsequently were found to be positive. The clinical history of recent travel to an endemic area and tick bite combined with the recent onset of multifocal erythema migrans lesions, systemic symptoms, elevated erythrocyte sedimentation rate, and positive Lyme serology supported the diagnosis of Lyme disease.

The appropriate clinical context and cutaneous morphology are key when considering the differential diagnosis for multifocal annular lesions. Several entities comprised the differential diagnosis considered in our patient. Sweet syndrome is a neutrophilic dermatosis that can present with fever and varying painful cutaneous lesions. It often is associated with certain medications, underlying illnesses, and infections. ${ }^{1}$ Our patient's lesions were not painful, and she had no notable medical history, recent infections, or new medication use, making Sweet syndrome unlikely. A fixed drug eruption was low on the differential, as the patient denied starting any new medications within the 3 months prior to presentation. Erythema multiforme is an acute-onset immunemediated condition of the skin and mucous membranes that typically affects young adults and often is associated with infection (eg, herpes simplex virus, Mycoplasma pneumoniae) or medication use. Cutaneous lesions typically are self-limited, less than $3 \mathrm{~cm}$ targets with 3 concentric distinct color zones, often with central bullae or erosions. Although erythema multiforme was higher on the differential, it was less likely, as the patient lacked mucosal lesions and did not have symptoms of underlying herpetic or mycoplasma infection, and the clinical picture was more consistent with Lyme disease. Lastly, the failure for individual skin lesions to resolve within 24 hours excluded the diagnosis of urticaria.

Lyme disease is a tick-borne illness caused by 3 species of the Borrelia spirochete: Borrelia burgdorferi, Borrelia afzelii, and Borrelia garinii. ${ }^{2}$ In the United States, the disease predominantly is caused by $B$ burgdorferi that is endemic in the upper Midwest and Northeast regions. ${ }^{3}$ There are 3 stages of Lyme disease: early localized, early disseminated, and late disseminated disease. Early localized disease typically presents with a characteristic single erythema migrans (EM) lesion 3 to 30 days following a bite by the tick Ixodes scapularis. ${ }^{2}$ The EM lesion gradually can enlarge over a period of several days, reaching up to 12 inches in diameter. ${ }^{2}$ Early disseminated disease can occur weeks to months following a tick bite and may present with systemic symptoms, multiple widespread
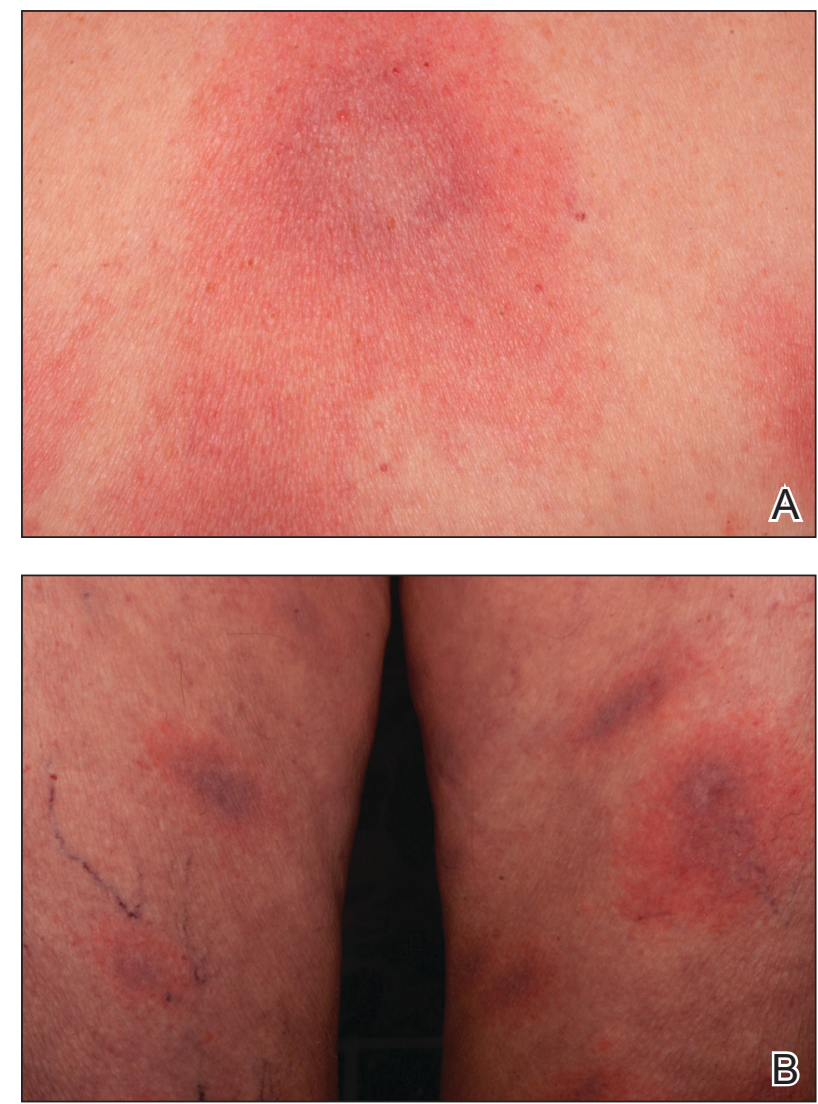

Disseminated erythema chronicum migrans. A and B, Multiple lesions were present on the abdomen and thighs.

EM lesions, neurologic features such as meningitis or facial nerve palsy, and/or cardiac manifestations such as atrioventricular block or myocarditis. Late disseminated disease can present with chronic arthritis or encephalopathy after months to years if the disease is left untreated. ${ }^{4}$

Early localized Lyme disease can be diagnosed clinically if the characteristic EM lesion is present in a patient who visited an endemic area. Laboratory testing and Lyme serology are neither required nor recommended in these cases, as the lesion often appears before adequate time has lapsed to develop an adaptive immune response to the organism. ${ }^{5}$ In contrast, Lyme serology should be ordered in any patient who meets all of the following criteria: (1) patient lives in or has recently traveled to an area endemic for Lyme disease, (2) presence of a risk factor for tick exposure, and (3) symptoms consistent with early disseminated or late Lyme disease. Patients with signs of early or late disseminated disease typically are seropositive, as IgM antibodies can be detected within 2 weeks of onset of the EM lesion and IgG antibodies within 2 to 
6 weeks. ${ }^{6}$ The Centers for Disease Control and Prevention recommends a 2-tiered approach when testing for Lyme disease. ${ }^{7}$ A screening test with high sensitivity such as an enzyme-linked immunosorbent assay or an immunofluorescence assay initially should be performed. ${ }^{7}$ If results of the screening test are equivocal or positive, secondary confirmatory testing should be performed via $\operatorname{IgM}$, with or without IgG Western immunoblot assay. ${ }^{7}$ Biopsy with histologic evaluation can reveal nonspecific findings of vascular endothelial injury and increased mucin deposition. Patients with suspected Lyme disease should immediately be started on empiric treatment with doxycycline $100 \mathrm{mg}$ twice daily for a minimum of 10 days (14-28 days if there is concern for dissemination) to prevent post-Lyme sequelae. ${ }^{5}$ Our patient's cutaneous lesions responded to oral doxycycline.

\section{REFERENCES}

1. Sweet's syndrome. Mayo Clinic. Accessed January 8, 2021. https://www.mayoclinic.org/diseases-conditions/sweets-syndrome /symptoms-causes/syc-20351117

2. Steere AC. Lyme disease. N Engl J Med. 2001;345:115-125.

3. Lyme disease maps: most recent year. Centers for Disease Control and Prevention. Updated November 22, 2019. Accessed January 8, 2021. https://www.cdc.gov/lyme/datasurveillance /maps-recent.html.

4. Steere AC, Sikand VK. The present manifestations of Lyme disease and the outcomes of treatment. N Engl J Med. 2003;348:2472-2474.

5. Sanchez E, Vannier E, Wormser GP, et al. Diagnosis, treatment, and prevention of Lyme disease, human granulocytic anaplasmosis, and babesiosis: a review. JAMA. 2016;315:1767-1777.

6. Shapiro ED. Borrelia burgdorferi (Lyme disease). Pediatr Rev. 2014; 35:500-509.

7. Mead P, Petersen J, Hinckley A. Updated CDC recommendation for serologic diagnosis of Lyme disease. MMWR Morb Mortal Wkly Rep. 2019;68:703.

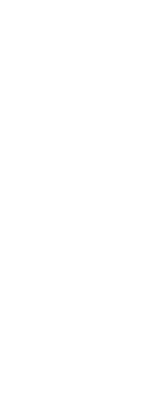

